\title{
Log canonical thresholds of divisors on Fano manifolds of Picard number 1
}

\author{
Jun-Muk Hwang
}

\begin{abstract}
Using a result of E. Viehweg, we study the behavior of the log canonical thresholds of divisors along free rational curves. This leads to a bound on the log canonical thresholds of divisors in terms of the degree of free rational curves on Fano manifolds of Picard number 1.
\end{abstract}

\section{Introduction}

For a point $y$ on a complex manifold $Y$ and an effective divisor $D$ on $Y$, the log canonical threshold $\operatorname{lct}_{y}(D)$ of $D$ at $y$ is defined by

$$
\operatorname{lct}_{y}(D):=\sup \left\{\text { rational number } c>0:|f|^{-2 c} \text { is locally integrable }\right\},
$$

where $f$ denotes the local defining function of $D$ at $y$. This is a very important local invariant of the divisor. See [Laz04, ch. 9] for other equivalent definitions and basic properties as well as many applications of log canonical thresholds.

One of the motivations for the current work is the following result of Ein and Lazarsfeld [EL97, 3.5].

Theorem 1. Let $(A, \Theta)$ be a principally polarized abelian variety. Then for any positive integer $k$ and $D \in|k \Theta|$,

$$
\operatorname{lct}_{x}(D) \geqslant \frac{1}{k} \quad \text { for each } x \in A \text {. }
$$

A principally polarized abelian variety is, among other things, a homogeneous projective variety with a natural choice of a line bundle. It is natural to ask whether an analog of Theorem 1 holds for other homogeneous projective varieties. In this paper we will prove the following analog of Theorem 1 for rational homogeneous spaces.

Theorem 2. Let $G / P$ be a rational homogeneous space of Picard number 1 and let $L$ be the ample generator of the Picard group of $G / P$. Then for any positive integer $k$ and $D \in|k L|$,

$$
\operatorname{lct}_{x}(D) \geqslant \frac{1}{k} \quad \text { for each } x \in G / P \text {. }
$$

As far as we know, this is new even when $k=1$. For Grassmannians, Theorem 2 was proved in [Hwa06]. The proof in [Hwa06] used Kapranov's work on the derived category of coherent sheaves on Grassmannians and vanishing theorems of Nadel and Demailly. This argument was modeled on that of [EL97], which used Mukai's work on the derived category of coherent sheaves on $A$ and Nadel's vanishing theorem. This method seems very hard to generalize to arbitrary $G / P$.

Received 18 February 2006, accepted in final form 16 June 2006.

2000 Mathematics Subject Classification 14J45.

Keywords: log canonical thresholds, multiplier ideal sheaf, free rational curve.

This journal is (C) Foundation Compositio Mathematica 2007. 


\section{J.-M. HWANG}

The method we will use in this paper is completely different. It is motivated by another problem. To explain this, let us recall the following well-known conjecture on the degree of Fano manifolds.

Conjecture 1. Let $X$ be a Fano manifold of Picard number 1. Then

$$
\left(-K_{X}\right)^{\operatorname{dim} X} \leqslant(\operatorname{dim} X+1)^{\operatorname{dim} X} .
$$

Moreover, $X$ is the projective space when the equality holds.

For $\operatorname{dim} X=3$, this follows from the classification of Fano threefolds (cf. [Isk80]). For $\operatorname{dim} X=4$, this was proved in [Hwa03]. It is open for $\operatorname{dim} X \geqslant 5$. A closely related problem is the following.

Conjecture 2. Let $X$ be a Fano manifold of Picard number 1 and $x \in X$ be a general point. Then for any positive integer $k$ and $D \in\left|-k K_{X}\right|$,

$$
\operatorname{mult}_{x}(D) \leqslant 2 k \operatorname{dim} X \text {. }
$$

The bound in Conjecture 2 is optimal and it is achieved when $X$ is a hyperquadric and $D$ is a multiple of a singular hyperplane section. Conjecture 2 implies a slightly weaker form of Conjecture 1 : by Riemann-Roch, it gives the bound

$$
\left(-K_{X}\right)^{\operatorname{dim} X} \leqslant(2 \operatorname{dim} X)^{\operatorname{dim} X} .
$$

In [RC00], Conjecture 2 was proved under the additional assumption that the tangent bundle of $X$ is semi-stable. The semi-stability of the tangent bundle of $X$ was verified when $\operatorname{dim} X \leqslant 6$ in [Hwa98]. In higher dimension, the following result was proved in [Cam91], [KMM92] and [Nad91].

Theorem 3. Let $X$ be a Fano manifold of Picard number 1 and $x \in X$ be a general point. Then for any positive integer $k$ and $D \in\left|-k K_{X}\right|$,

$$
\operatorname{mult}_{x}(D) \leqslant k(\operatorname{dim} X) \cdot(\operatorname{dim} X+1) .
$$

In this paper, we will prove the following, which can be regarded as half-way between Conjecture 2 and Theorem 3.

Theorem 4. Let $X$ be a Fano manifold of Picard number 1 and $x \in X$ be a general point. Then for any positive integer $k$ and $D \in\left|-k K_{X}\right|$,

$$
\operatorname{lct}_{x}(D) \geqslant \frac{1}{k(\operatorname{dim} X+1)} .
$$

Moreover, if the equality holds for some $k$ and $D$, then $X$ is the projective space.

In fact, there is a well-known relation (cf. $[\operatorname{Laz04}, 9.3 . \mathrm{A}])$ between $\operatorname{mult}_{x}(D)$ and $\operatorname{lct}_{x}(D)$ given by the inequalities

$$
\frac{1}{\operatorname{mult}_{x}(D)} \leqslant \operatorname{lct}_{x}(D) \leqslant \frac{\operatorname{dim} X}{\operatorname{mult}_{x}(D)} .
$$

These inequalities show that Theorem 4 implies Theorem 3, while Conjecture 2 implies a slightly weaker form of Theorem 4, namely,

$$
\operatorname{lct}_{x}(D) \geqslant \frac{1}{2 k(\operatorname{dim} X)} \text { for each } x \in X .
$$

The fact that Conjecture 2 implies both Conjecture 1 and Theorem 4 in the weaker form where $\operatorname{dim} X+1$ is replaced by $2 \operatorname{dim} X$, seems to suggest that there may be a direct connection between Conjecture 1 and Theorem 4. However, so far we are unable to find such a direct relation.

Both Theorem 2 and Theorem 4 are simple consequences of Theorem 5 below. Recall that a rational curve $C$ on a compact complex manifold $X$ is free if, under the normalization $f: \mathbf{P}_{1} \rightarrow$ $C \subset X$, the pull-back $f^{*} T_{X}$ of the tangent bundle of $X$ is numerically effective (nef). 


\section{LOG CANONICAL THRESHOLDS OF DIVISORS}

Theorem 5. Let $X$ be a Fano manifold of Picard number 1 and $C \subset X$ be a free rational curve. Then there exists a Zariski dense open subset $U \subset X$ determined by $C$ such that, for any effective divisor $D$ on $X$ and $x \in U$,

$$
\operatorname{lct}_{x}(D) \geqslant \frac{1}{C \cdot D}
$$

Theorem 5 is a consequence of the following more general result, combined with some well-known facts about the geometry of free rational curves on Fano manifolds of Picard number 1.

Theorem 6. Let $X$ be a compact complex manifold and $C \subset X$ be a free rational curve. For any effective divisor $D$ on $X$, either $\operatorname{lct}_{x}(D) \geqslant 1 /(C \cdot D)$ for each $x \in C$, or $\operatorname{lct}_{x}(D)=\operatorname{lct}_{x^{\prime}}(D)$ for any two points $x, x^{\prime} \in C$.

The proof of Theorem 6 uses a result about log canonical thresholds, which we call the 'product theorem' and explain in $\S 2$. This result is just a slight variation of an old result of Viehweg in [Vie95]. In fact, the proof of [Vie95, Proposition 5.19] works verbatim to give our product theorem. The only difference is that Viehweg stated his result in terms of his invariant $e(D)$ in place of log canonical thresholds. His invariant $e(D)$ is different from the log canonical thresholds, but it is of the same nature. While the log canonical threshold is defined as the supremum of rational numbers $c$ making $|f|^{-2 c}$ integrable, Viehweg's invariant is defined as the minimal positive integer $N$ making $|f|^{-1 / N}$ integrable. Thus our contribution on the product theorem is just to formulate it in terms of log canonical thresholds and highlight it by giving applications to Fano manifolds.

The name 'product theorem' originates from Nadel's 'product theorem' in [Nad91, 2.4, 2.5], which is the essential point in his proof of Theorem 3. In this sense, our proof of Theorem 4 follows the line of thought of [Nad91]. Nadel's product theorem is about the behavior of multiplicities of a divisor on a fibered space along a fiber. Our product theorem is concerned with the behavior of the log canonical thresholds in a similar setting. However their proofs are essentially different. Also, it is rather remarkable that our product theorem gives the optimal bound for log canonical thresholds in Theorem 4, while Nadel's, up to now, cannot give the optimal bound for multiplicities in Conjecture 2.

\section{Product theorem}

Given a line bundle $L$ on a compact complex manifold $Z$ with $|L| \neq \emptyset$, define

$$
\operatorname{lct}(L):=\inf _{E \in|L|, z \in Z} \operatorname{lct}_{z}(E) .
$$

Product theorem. Let $f: X \rightarrow Y$ be a smooth projective morphism between two complex manifolds. Let $y \in Y$ be a point and $X_{y}=f^{-1}(y)$ be the fiber over $y$. Let $D$ be an effective divisor on $X$ and let $L$ be the restriction of the line bundle $\mathcal{O}_{X}(D)$ to $X_{y}$. Then either $\operatorname{lct}_{x}(D) \geqslant \operatorname{lct}(L)$ for each $x \in X_{y}$ or $\operatorname{lct}_{x_{1}}(D)=\operatorname{lct}_{x_{2}}(D)$ for any two points $x_{1}, x_{2} \in X_{y}$.

As mentioned in the introduction, this is essentially proved by Viehweg in [Vie95, Proposition 5.19]. However, he formulated it in terms of his invariant $e(D)$, which is different from log canonical thresholds. Moreover, the terms used in [Vie95] do not seem to be widely known. For the reader's convenience, we will rewrite the proof in the language of [Laz04].

To start with, recall that the multiplier ideal sheaf of an effective Q-divisor $D$ on a complex manifold $X$ is the ideal sheaf $\mathcal{J}(D)$ defined by

$$
\mathcal{J}(D):=\left\{g \in \mathcal{O}_{X}:|g|^{2} \cdot|f|^{-2} \text { is locally integrable }\right\},
$$




\section{J.-M. HWANG}

where $f$ is the multi-valued local holomorphic function defining $D$. Thus for an effective divisor $D$ and $x \in X$,

$$
\operatorname{lct}_{x}(D)=\sup \left\{c \in \mathbf{Q}: \mathcal{J}(c D)_{x}=\mathcal{O}_{x}\right\} .
$$

We will use basic properties of multiplier ideal sheaves presented in [Laz04, ch. 9]. It is interesting to note that many important results about multiplier ideal sheaves already appeared in [Vie95], at least implicitly.

Lemma 1. Let $f: X \rightarrow Y$ be a smooth projective morphism between two complex manifolds. Let $y \in Y$ be a point and $X_{y}=f^{-1}(y)$ be the fiber over $y$. Let $\Gamma$ be an effective divisor on $X$ whose support does not contain $X_{y}$. Let $\Delta \subset Y$ be an effective Q-divisor with normal crossing support. If $c<\operatorname{lct}_{x}\left(\left.\Gamma\right|_{X_{y}}\right)$ for all $x \in X_{y}$, then

$$
\mathcal{J}\left(c \Gamma+f^{*} \Delta\right)=\mathcal{O}_{X}\left(-f^{*}[\Delta]\right)
$$

in a neighborhood of $X_{y}$.

Proof. If $\Delta=0$, this follows from the behavior of multiplier ideal sheaves under restriction [Laz04, 9.5.1]. When $\Delta \neq 0$, replacing $[\Delta]$ by $\Delta-[\Delta]$, we may assume that the round-down $[\Delta]$ is 0 . Let $Y_{1}$ be an irreducible component of $\Delta$ with multiplicity $\mu<1$. Define $\Delta^{\prime}:=\Delta-\mu Y_{1}$. Let $X_{1}:=f^{-1}\left(Y_{1}\right)$ and consider $f_{1}:=\left.f\right|_{X_{1}}: X_{1} \rightarrow Y_{1}$. By induction on dimension, we can assume that Lemma 1 holds for the morphism $f_{1}$, the divisor $\left.\Gamma\right|_{X_{1}}$ and the Q-divisor $\left.\Delta^{\prime}\right|_{Y_{1}}$ with normal crossing support. Thus we have $\mathcal{J}\left(\left.\left(c \Gamma+f^{*} \Delta^{\prime}\right)\right|_{X_{1}}\right)=\mathcal{O}_{X_{1}}$ in a neighborhood of $X_{y}$ in $X_{1}$. Then by the inversion of adjunction [Laz04, 9.5.11],

$$
\mathcal{J}\left(c \Gamma+f^{*} \Delta\right)=\mathcal{J}\left(c \Gamma+f^{*} \Delta^{\prime}+\mu X_{1}\right)=\mathcal{O}_{X}
$$

in a neighborhood of $X_{y}$ in $X$.

The next lemma is precisely [Vie95, Claim 5.20]. We will omit the proof.

Lemma 2. Let $f: X \rightarrow Y$ be a smooth projective morphism between complex manifolds and $D$ be an effective divisor on $X$. Then there exists a smooth modification $\delta: Y^{\prime} \rightarrow Y$ and an effective normal crossing divisor $\Delta$ on $Y^{\prime}$ such that on the fiber product



the divisor $\Gamma:=\eta^{*} D-g^{*} \Delta$ is effective and does not contain any fiber of $g$.

Lemma 3. Let $f: X \rightarrow Y$ and $D$ be as in the product theorem. If $c<\operatorname{lct}(L)$ and $\mathcal{J}(c D)_{x_{1}}=\mathcal{O}_{x_{1}}$ for some $x_{1} \in X_{y}$, then $\mathcal{J}(c D)_{x_{2}}=\mathcal{O}_{x_{2}}$ for any $x_{2} \in X_{y}$.

Proof. Let us use the notation of Lemma 2. Since $c<\operatorname{lct}(L)$, we have $c<\operatorname{lct}\left(\left.\Gamma\right|_{X_{y^{\prime}}^{\prime}}\right)$ for each $y^{\prime} \in \delta^{-1}(y)$. Thus by Lemma 1 ,

$$
\mathcal{J}\left(\eta^{*}(c D)\right)=\mathcal{J}\left(c \Gamma+g^{*}(c \Delta)\right)=\mathcal{O}_{X^{\prime}}\left(-g^{*}[c \Delta]\right)
$$

in a neighborhood of $X_{y^{\prime}}^{\prime}$ for each $y^{\prime} \in \delta^{-1}(y)$, thus in a neighborhood of $\eta^{-1}\left(X_{y}\right)$. By the birational transformation rule for multiplier ideals in [Laz04, 9.2.33],

$$
\mathcal{J}(c D)=\eta_{*}\left(\mathcal{J}\left(\eta^{*}(c D)\right) \otimes \mathcal{O}_{X^{\prime}}\left(K_{X^{\prime} / X}\right)\right)=\eta_{*}\left(\mathcal{O}_{X^{\prime}}\left(K_{X^{\prime} / X}-g^{*}[c \Delta]\right)\right)
$$

in a neighborhood of $X_{y}$. Since $\eta$ is induced by the fiber product, the stalks of the sheaf on the right hand side along a fiber of $f$ cannot vary. This proves Lemma 3. 


\section{LOG CANONICAL THRESHOLDS OF DIVISORS}

Proof of the product theorem. Pick $x_{2} \in X_{y}$ such that $\operatorname{lct}_{x}(D) \geqslant \operatorname{lct}_{x_{2}}(D)$ for all $x \in X_{y}$. Suppose there exists $x_{1} \in X_{y}$ such that $\operatorname{lct}_{x_{1}}(D)>\operatorname{lct}_{x_{2}}(D)$. Let $c$ be any positive rational number satisfying

$$
\operatorname{lct}_{x_{1}}(D)>c>\operatorname{lct}_{x_{2}}(D) .
$$

Then Lemma 3 says that $c \geqslant \operatorname{lct}(L)$. Since this is true for any choice of such $c$, we conclude that $\operatorname{lct}_{x_{2}}(D) \geqslant \operatorname{lct}(L)$.

Corollary 1. Let $T$ be a complex manifold and $D$ be an effective divisor in $T \times \mathbf{P}_{1}$ such that, for a general point $t \in T, D$ has intersection number $d$ with the curve $\{t\} \times \mathbf{P}_{1}$. Then for any $t_{o} \in T$, either $\operatorname{lct}_{x}(D) \geqslant 1 / d$ for each $x \in\left\{t_{o}\right\} \times \mathbf{P}_{1}$, or $\operatorname{lct}_{x}(D)=\operatorname{lct}_{x^{\prime}}(D)$ for any two points $x, x^{\prime} \in\left\{t_{o}\right\} \times \mathbf{P}_{1}$.

Proof. It suffices to note that if $X$ is a non-singular projective curve and $L$ is a line bundle with $|L| \neq \emptyset$, then $\operatorname{lct}(L)=1 / \operatorname{deg} L$.

\section{Proofs of theorems}

Proof of Theorem 6. Let $\operatorname{Hom}^{\text {free }}\left(\mathbf{P}_{1}, X\right)$ be the space of free morphisms and

$$
F: \mathbf{P}_{1} \times \operatorname{Hom}^{\text {free }}\left(\mathbf{P}_{1}, X\right) \rightarrow X
$$

be the evaluation morphism, as in [Kol96, II.3.5.4]. By [Kol96, II.3.5.4], $\operatorname{Hom}^{\text {free }}\left(\mathbf{P}_{1}, X\right)$ is nonsingular and $F$ is a smooth morphism. This implies, by [Laz04, 9.5.45], that, for each $u \in \mathbf{P}_{1} \times$ Hom $^{\text {free }}$,

$$
\operatorname{lct}_{u}\left(F^{*} D\right)=\operatorname{lct}_{F(u)}(D) .
$$

Thus Theorem 6 is a direct consequence of Corollary 1 applied to $T=\operatorname{Hom}^{\text {free }}\left(\mathbf{P}_{1}, X\right)$.

Proof of Theorem 5. Denote by RatCurves ${ }^{n}(X)$ the space of rational curves on $X$, following [Kol96, II.2]. Let $\mathcal{K}$ be a component of $\operatorname{RatCurves}^{n}(X)$ to which $C$ belongs. By [Kol96, IV.4.14] applied to the family $\mathbf{P}_{1} \times \mathcal{K} \rightarrow \mathcal{K}$, there exists an open set $W \subset X \times X$ such that if $\left(x_{1}, x_{2}\right) \in W$, then $x_{1}$ and $x_{2}$ can be connected by a connected chain of free rational curves belonging to $\mathcal{K}$. Then we choose $U \subset X$ as a Zariski open subset in the image of the projection of $W$ to the first factor. Suppose that there exists a point $x_{1} \in U$ with $\operatorname{lct}_{x_{1}}(D)<1 /(C \cdot D)$. We can choose a point $x_{2} \notin D$ such that $x_{1}$ and $x_{2}$ can be connected by a connected chain of free rational curves belonging to $\mathcal{K}$. Applying Theorem 6 repeatedly, we get $\operatorname{lct}_{x_{2}}(D)=\operatorname{lct}_{x_{1}}(D)<1 /(C \cdot D)$, a contradiction.

Proof of Theorem 4. By [Kol96, IV.2.10], there exists a free rational curve $C$ satisfying $C \cdot\left(-K_{X}\right) \leqslant$ $\operatorname{dim} X+1$. Thus the inequality in Theorem 4 follows from Theorem 5 . On the other hand, if $X$ is different from the projective space, [CMS02] says that there exists a free rational curve $C$ with $C \cdot\left(-K_{X}\right) \leqslant \operatorname{dim} X$. Thus the equality in Theorem 4 cannot hold.

Proof of Theorem 2. When $X=G / P$, there exists a free rational curve $C$ satisfying $C \cdot L=1$ (e.g. [Kol96, V.1.15]). Thus Theorem 2 follows from Theorem 5 and the homogeneity of $X$.

\section{ACKNOWLEDGEMENTS}

I would like to thank Lawrence Ein, Rob Lazarsfeld and Yum-Tong Siu for valuable suggestions and encouragement. I am particularly grateful to Eckart Viehweg for pointing out to me that the product theorem, for which I initially gave a messy analytic argument, can be proved as his Proposition 5.19 in [Vie95]. 


\section{LOG CANONICAL THRESHOLDS OF DIVISORS}

\section{REFERENCES}

Cam91 F. Campana, Une version géométrique généralisée du théorème du produit de Nadel, Bull. Soc. Math. France 119 (1991), 479-493.

CMS02 K. Cho, Y. Miyaoka and N. I. Shepherd-Barron, Characterizations of projective space and applications to complex symplectic manifolds, in Higher dimensional birational geometry, Kyoto, 1997, Advanced Studies in Pure Mathematics, vol. 35 (Mathematical Society of Japan, Tokyo, 2002), 1-88.

EL97 L. Ein and R. Lazarsfeld, Singularities of theta divisors and the birational geometry of irregular varieties, J. Amer. Math. Soc. 10 (1997), 243-258.

Hwa98 J.-M. Hwang, Stability of tangent bundles of low dimensional Fano manifolds with Picard number 1, Math. Ann. 312 (1998), 599-606.

Hwa03 J.-M. Hwang, On the degrees of Fano four-folds of Picard number 1, J. reine angew. Math. 556 (2003), 225-235.

Hwa06 J.-M. Hwang, Log canonical thresholds of divisors on Grassmannians, Math. Ann. 334 (2006), 413-418.

Isk80 V. A. Iskovskikh, Anticanonical models of 3-dimensional algebraic varieties, J. Soviet Math. 13 (1980), 745-814.

Kol96 J. Kollár, Rational curves on algebraic varieties, Ergebnisse der Mathematik und ihrer Grenzgebiete (3), vol. 32 (Springer, Berlin, 1996).

KMM92 J. Kollár, Y. Miyaoka and S. Mori, Rational curves on Fano varieties, in Proc. Algebraic Geometry Conf., Trento, Lecture Notes in Mathematics, vol. 1515 (Springer, Berlin, 1992), 100-105.

Laz04 R. Lazarsfeld, Positivity in algebraic geometry. II. Positivity for vector bundles, and multiplier ideals, Ergebnisse der Mathematik und ihrer Grenzgebiete (3), vol. 49 (Springer, Berlin, 2004).

Nad91 A. Nadel, The boundedness of degree of Fano varieties with Picard number one, J. Amer. Math. Soc. 4 (1991), 681-692.

RC00 Z. Ran and H. Clemens, A new method in Fano geometry, Int. Math. Res. Not. 2000, 527-549.

Vie95 E. Viehweg, Quasi-projective moduli for polarized manifolds, Ergebnisse der Mathematik und ihrer Grenzgebiete (3), vol. 30 (Springer, Berlin, 1995).

Jun-Muk Hwang jmhwang@kias.re.kr

Korea Institute for Advanced Study, 207-43 Cheongryangri-dong, Seoul 130-722, Korea 